\title{
Effectiveness of Deep Breathing Exercises and Incentive Spirometry on Arterial Blood Gases in Second Degree Inhalation Burn Patients
}

\author{
Summyia Siddique Malik and Naureen Tassadaq \\ Foundation University Institute of Rehabilitation Sciences, Islamabad, Pakistan
}

\begin{abstract}
Objective: To find out the effectiveness of the deep breathing exercises and incentive spirometryon arterial blood gases in second degree inhalation burn patients.

Study Design: Randomised control trial.

Place and Duration of Study: The study was conducted in the Burns Unit of PIMS Hospital, Islamabad from September 2017 to March 2018.

Methodology: The data was collected on structured questionnaire using respiratory distress scoring and objective tools including arterial blood gases (ABGs) and vital signs with signed consent. There was random allocation of patients in the control and experimental groups. Baseline data was collected on day 00 and after completion of 14 sessions, it was recollected on day 07 , and was assessed by using independent t-test and paired t-test. Both groups were given standard medical and nursing care. The experimental group was given single treatment regimen i.e. deep breathing exercises (given twice daily with 5-10 repetitions of each DBE possible onto patient for a period of 15-30 minutes). ISM was being performed on control group patients with 10-15 cycles along with steam inhalation and nebulization with beta 2 agonist for a period of 15-20 minutes for 35-45 minutes twice daily for a period of 07 days.

Results: The male to female ratio was 7:8 in control group and 8:7 in the experimental group. The mean age was 29.6 \pm 6.66 years in the control group, and $38.4 \pm 4.91$ years in the experimental group. The $\mathrm{pH}$ in arterial blood gas analysis of the experimental group was $7.33(p=0.002)>\mathrm{pO}_{2}$ was $90.53(p<0.001)$ and $\mathrm{pCO} 2$ was $41.61(p=0.626)$. For control group, the $\mathrm{pH}$ in $\mathrm{ABGs}$ was $7.36(\mathrm{p}=0.268), \mathrm{pO} 2$ was $88.06(\mathrm{p}=0.739)$ and $\mathrm{pCO}_{2}$ was $38.86(\mathrm{p}<0.001)$.

Conclusion: Deep breathing exercises are significant in improving pulmonary complications in patients of second degree inhalation burns.
\end{abstract}

Key Words: Respiratory distress scoring, Incentive spirometry, Arterial blood gases.

How to cite this article: Malik SS, Tassadaq N. Effectiveness of deep breathing exercises and incentive spirometry on arterial blood gases in second degree inhalation burn patients. J Coll Physicians Surg Pak 2019; 29(10):954-7.

\section{INTRODUCTION}

Burn injuries pose a high burden of fatalities on the health systems worldwide. About $90 \%$ of burn incidents occur in low to middle income countries, areas that are deficient in the essential means to decrease the rate and intensity of such injuries. ${ }^{1}$ Respiratory injury, resulting from inhalation of smoke or chemical products of combustion, is associated with significant morbidity and mortality either directly or due to post-burn respiratory complications like pneumonia. ${ }^{2}$ In addition to early mobilisation and change of position, a variety of deep breathing exercises and coughing techniques are also beneficial in this regard. ${ }^{3}$ Significant work has been done

Correspondence to: Summyia Siddique Malik, Foundation University Institute of Rehabilitation Sciences, Islamabad, Pakistan

E-mail: Physiotherapy19@gmail.com

Received: August 11, 2018; Revised: July 06, 2019;

Accepted: July 09, 2019 in early 1900's about medical and nursing care for burned patients. ${ }^{4}$ The increase in mortality of the burned patient is primarily due to post-burn respiratory complications like emphysema, pneumonia rather due to burn trauma.

The air passage is most important in not only gas exchange but also in exchange of heat. Smoke injury affects the entire respiratory tract. ${ }^{5}$ In injuries caused by inhalation of smoke, there is the deposition of two toxic acids that are being produced as a result of chemical interaction among sulfur dioxide and nitrogen dioxide, which adds up water from humidity created in the cell thus resulting in an additional acid burn in already affected areas. There is more resistance due to swelling of the burned structures. ${ }^{6}$

Spirometer was found to be effective in reducing length of hospital stay. ${ }^{7}$ Spirometer is a device used to increase trans-pulmonary pressure and inspiratory volumes, improve inspiratory muscle performance, and reestablish or simulate the normal pattern of pulmonary hyperinflation in the patients suffering from pulmonary 
complications. Suman and his coworkers found that respiratory management of pulmonary injury by using standard nursing care along with chest physiotherapy involving percussion is less effective as compared to deep breathing exercises performed onto burn-patients. ${ }^{8}$ Jeschke et al. did a remarkable work on respiratory management of inhalation injury by use of both percussion and deep breathing exercises in clearing trachea bronchial tree. ${ }^{9}$ Chen et al. conducted a survey on the current status of burn rehabilitation services in China, showing that even comprehensive cardiopulmonary rehabilitation of burn patients is required as it is negligible. 10

In Pakistan, post-burn rehabilitation is practised only in few places with well-versed burn physical therapists available. The mortality rate in these burn patients is high due to respiratory complications and mismanagement of these complications. This study was conducted to find out the best effective method for preventing postburn respiratory complications.

\section{METHODOLOGY}

It was a randomised control trial conducted at PIMS Hospital, Islamabad, from September 2017 to March 2018. Sample size was calculated as 30 , using open Epi with $95 \%$ confidence interval, $5 \%$ margin of error and $50 \%$ response distribution. Patients were randomly allocated using lottery method in control group and experimental group, with 15 patients in each group. Blinding was ensured. Sampling was done using nonprobability convenient sampling technique. Treatment session was given twice a day regularly for a period of 07 consecutive days.

Data was collected twice; baseline data was collected on day 0 ; and after 14 sessions on day 07 ; data was recollected.

All the patients between 20-50 years, with second degree inhalation burn, after their first surgical intervention, using nasal canula and face mask for oxygen support were included without gender discrimination. The exercises were initiated after first surgical intervention with mostly 3-4 days. However, unconscious patients with pre-burn comorbidities, suffering from other types of burns like chemical burns, and who were on ventilator support were excluded from the study.

This study was initiated after approval from advanced study \& research committee (ASRC) of Isra Institute of Rehabilitation Sciences (IIRS). The study was conducted according to the ethical guidelines of Pakistan Medical Research Council (PMRC) and the Declaration of Helsinki. Anonymity and confidentiality of the participants were maintained throughout the research. Informed consent was obtained from all the study participants/ attendants, prior to recruiting in the study. The data was collected on self-structured questionnaire. The treatment of experimental group was based on single physical therapy treatment regimen that is deep breathing exercises (DBE) only along with standard medical and nursing care. A qualified and trained physical therapist helped the patient performing these exercises. The patients performed 4-6 repetitions of each DBE with session of 5-10 minutes daily. Patients in this group were given deep breathing exercises involving belly breathing. For this, patients were asked to lie on flat surface with hands on lower ribs. Then patients were asked to breathe in deeply and focus on movements of hands over lower ribs. Then patients were allowed to exhale forcefully until the abdomen was back to its starting position. Then the patients were asked to repeat this pattern. Natural breathing technique was initiated with patients in their resting supine position, the patients were then asked to inhale using nose while noticing the movement of abdomen. In order to fill the lungs to their maximum capacity, the patients were asked to breathe by successively expanding abdomen and then holding the breath before complete exhalation. Before the initiation of the session, a brief demonstration of the exercise was given to the patients in order to initiate active cycle of breathing technique. The patients were taught breathing control followed by 2-3 deep breaths along with low volume huffs, later on followed by high volume huff and then an effective cough. Blowing the candles was the caution given to the patients for pursed lip breathing technique. The patients were given a tactile stimulation on lower ribs followed by the caution of inflating lower ribs in order to teach diaphragmatic breathing technique. These were performed according to the patients' cooperation.

The members of control group were given 5-6 cycles of ISM with prior steam inhalation and nebulization as per treatment criteria, this session lasted for 20-30 minutes daily. The patients in control group were given a session of nebulization along with steam inhalation before spirometry. Likewise, patients were made to perform spirometry with 5-6 cycles twice a day every day and for a period of 07 days. Regarding spirometry, each patient was given incentive spirometer and was guided to carry the spirometer in hand in an upright position. Then patients were guided to exhale normally and then placing the lips tightly around the mouthpiece. Next step was to raise the ball in the chamber by a slow inhalation and sustained inflation for at least five seconds. At maximum inhalation, the mouthpiece was removed followed by a breath-hold and normal exhalation. The control group members were not at any kind of stake after been given only deep breathing exercises. A brief self-structured questionnaire was newly developed by using general demographics and arterial blood gas analysis as a tool. Each question had four options for each statement. With signed consent, a total of 17 questions were filled by the physical therapist at day 0 
Table I: Group comparison (Experimental n=15, Control n=15)

\begin{tabular}{|c|c|c|c|c|c|}
\hline Variables & Groups & $\mathrm{N}$ & Mean $(m)$ & Standard Deviation & $\mathrm{p}$-value \\
\hline \multirow[t]{2}{*}{ pH Pre-session } & Control & 15 & 7.05 & 1.03 & 0.396 \\
\hline & Experimental & 15 & 7.13 & 0.25 & \\
\hline \multirow[t]{2}{*}{ pHPost-session } & Control & 15 & 7.36 & 0.01 & 0.546 \\
\hline & Experimental & 15 & 7.33 & 0.09 & \\
\hline \multirow[t]{2}{*}{ pO2Pre-session } & Control & 15 & 86.00 & 0.92 & 0.694 \\
\hline & Experimental & 15 & 85.26 & 4.55 & \\
\hline \multirow[t]{2}{*}{ pO2Post-session } & Control & 15 & 88.06 & 23.85 & 0.213 \\
\hline & Experimental & 15 & 90.53 & 2.55 & \\
\hline \multirow[t]{2}{*}{ pCO2Pre-session } & Control & 15 & 46.60 & 0.98 & $0.006^{*}$ \\
\hline & Experimental & 15 & 43.06 & 10.69 & \\
\hline \multirow[t]{2}{*}{ pCO2Post-session } & Control & 15 & 38.86 & 1.59 & $0.054^{*}$ \\
\hline & Experimental & 15 & 41.61 & 3.18 & \\
\hline
\end{tabular}

and day 07 , respectively. The revised version of questionnaire is attached as supplementary file.

The data was collected by filling the questionnaire; it was paper and pen method. The data was then analysed by using SPSS version 21. Normality of data was checked using Shipore-Wilk test. Categorical variables were presented in the form of frequencies and percentages, whereas, continuous variables were presented in the form of mean \pm S.D. Statistical significance between groups was assessed using independent t-test. For within the group comparison, paired t-test was used. Statistical significance was assumed at $p$-value $<0.05$.

\section{RESULTS}

A total of $46 \%(07)$ males and $53 \%(08)$ females were in control group. However, 53\% (08) males and 46\% (07) females were in experimental group. The results showed a mean GCS of $13.6 \pm 1.11$ for control group and 14.2 \pm 1.08 for experimental group patients. The mean duration of exposure for control group was found to be $10.2 \pm 5.2$ seconds and for experimental group $9.7 \pm 3.8$ seconds.

The normal perceived ranges for arterial blood gases were as follows: $\mathrm{pH}=7.35-7.45$, for $\mathrm{pO}_{2}=88 \mathrm{mmHg}$ $100 \mathrm{mmHg}$ and for $\mathrm{pCO}_{2} 45 \mathrm{mmHg}-55 \mathrm{mmHg}$. The within group comparison results for control group were $p<0.001^{* * *}$ for $\mathrm{pCO} 2$ factor of arterial blood gases, $\mathrm{p}=0.268$ for $\mathrm{pH}$ and $\mathrm{p}=0.739$ for $\mathrm{pO}_{2}$ with $\mathrm{pCO}_{2}$ showing significant improvement $\mathrm{pH}$ and $\mathrm{pO}_{2}$ showing nonsignificant. The within-group comparison for experimental group were significant for $\mathrm{pH}$ and $\mathrm{pO}_{2}$, with $\mathrm{p}=0.002^{* *}$ for $\mathrm{pH}, \mathrm{p}<0.001^{* * *}$ for $\mathrm{pO}_{2}$ and $\mathrm{p}=0.626$ for $\mathrm{pCO}_{2}$ (Table I).

\section{DISCUSSION}

Deep breathing exercises are more effective in improving arterial blood gases of patients suffering from second degree inhalation burns with $p=.002$ for $p H$ and $p<0.001$ for $\mathrm{pO} 2$ as compared to control group. However, between groups comparisons showed that both treatments are equally effective.

Currently, there are very few burn centres in Pakistan. In twin cities of Rawalpindi-Islamabad, only three to four burn centres are active and responsive. The current situation of chest rehabilitation in burn centre is not very good. The general attitude of surgeons towards rehabilitation is important as they ensure the physical therapy of each and every patient in burn ward.

After second degree inhalation burn, the main reason of post-burn mortality and morbidity is pulmonary complications, mainly pneumonia. ${ }^{2}$ So, there must be emphasis to prevent complications in early post-burn period. Incentive Spirometry is a respiratory therapy procedure that is still in use by many burn physical therapists and nurses. In this procedure a device (spirometer) is used to prevent and lessen post-burn complications in patients of second degree inhalational burn, immediately after hospital admittance. ${ }^{11}$ Present study was conducted to evaluate the efficacy of deep breathing exercises versus the normal conventional spirometry.

The results for post-session values of both experimental and control groups shown in Table I. The $\mathrm{pH}$ and $\mathrm{pO} 2$ changes were non-significant for both control and experimental groups. However, $\mathrm{pCO}_{2}$ changes were significant. The results showed that there was a significant decrease in $\mathrm{pCO}_{2}$ factor of $A B G s$ after continuing this treatment protocol for 14 sessions. However, there was no significant improvement seen in values of $\mathrm{pH}$ and $\mathrm{pO}_{2}$.

Dellinger et al. found that pulmonary complications caused or contributed to the death of most patients who die after thermal injury. Twenty-two out of 100 patients in that series developed pulmonary complications with $86 \%$ mortality. A programme of management, based on this review, is presented emphasising early intubation, mechanical ventilation for specific conditions, pulmonary artery pressure monitoring and no crystalloid fluid infusion. An improvement in mortality statistics has been noted since this programme was instituted. ${ }^{12}$ A study compared the aim of burn rehabilitation treatment with routine burn rehabilitation treatment to find out rehabilitation related problems.

The results of this study indicated a significant difference $(p<0.01)$ in burn contractures between two groups, that 
intensive burn rehabilitation decreased burn complications. BRT could consider BRT protocols. ${ }^{13}$ Chest physiotherapy now involves gravity-assisted bronchial drainage with chest percussion and vibrations. Studies have shown that a combination of techniques were effective in secretion removal. Bronchial drainage/ positioning is a therapeutic modality which uses gravityassisted positioning designed to improve pulmonary hygiene in patients with inhalation injury or retained secretions, percussion aids in the removal of secretions from the tracheal bronchial tree.

Major problems faced during rehabilitation of burn patients include delayed incorporation of burn rehabilitation which further leads to pulmonary infections. This further increases rate of contracture formation and; hence, increases the morbidity and mortality. ${ }^{14}$

Yang et al. conducted a research to assess the effectiveness and safety of chest physiotherapy for pneumonia in adults. 15 This systematic review showed that chest physical therapy can be used as an adjunct treatment.

The limitations in statistical significance are mainly due to the small sample size of the study. This can be overcome by conducting a multi-centered study.

\section{CONCLUSION}

Overall, both treatments are equally effective in improving arterial blood gases of patients suffering from second degree inhalation burns; however, deep breathing exercises are specifically more effective in improving arterial blood gases of patients suffering from second degree inhalation burns.

\section{ETHICAL APPROVAL:}

An ethical approval was taken from Ethical Review Board of Shaheed Zulfiqar Ali Bhutto Medical University, PIMS Hospital, Islamabad.

\section{PATIENTS' CONSENT:}

Informed consents were obtained in local language as per ethical guidelines.

\section{CONFLICT OF INTEREST:}

Authors declared no conflict of interest.

\section{AUTHORS' CONTRIBUTION:}

SSM: Conception of idea, data collection, leading role in manuscript writeup, verified the analytical process and contributed towards discussion.

NT: Critical feedback and discussion write up.

\section{REFERENCES}

1. Chan $\mathrm{CH}$, Yang SF, Yeh HW, Yeh YT, Wang YH, Teng YH, et al. Risk of pneumonia in patients with burn injury: A populationbased cohort study. Clin Epidemiol 2018; 10:1083-91.

2. Edelman DA, Khan N, Kempf K, White MT. Pneumonia after inhalation injury. J Burn Care Res 2007; 28:241-6.

3. Hantson P, Butera R, Clemessy JL, Michel A, Baud FJ. Early complications and value of initial clinical and paraclinical observations in victims of smoke inhalation without burns. Chest 1997; 111:671-5.

4. Barrow RE, Przkora R, Hawkins HK, Barrow LN, Jeschke MG, Herndon DN. Mortality related to gender, age, sepsis, and ethnicity in severely burned children. Shock 2005; 23:485-7.

5. Jones SW, Williams FN, Cairns BA, Cartotto R. Inhalation injury: Pathophysiology, diagnosis, and treatment. Clin Plast Surg 2017; 44:505-11.

6. Miller K, Chang A. Acute inhalation injury. Emerg Med Clin North Am 2003; 21:533-57.

7. Krivchenia K, Tumin D, Nemastil CJ, Tobias JD, Hayes D. Timing of spirometry may impact hospital length of stay for cystic fibrosis pulmonary exacerbation. Lung 2018; 196:207-11.

8. Mlcak RP, Suman OE, Herndon DN. Respiratory management of inhalation injury. Burns 2007; 33:2-13.

9. Jeschke MG, Chinkes DL, Finnerty CC, Kulp G, Suman OE, Norbury WB, et al. The pathophysiologic response to severe burn injury. Ann Surg 2008; 248:387-401.

10. Chen J, Li-Tsang CW, Yan H, Liang G, Tan J, Yang S, et al. A survey on the current status of burn rehabilitation services in China. Burns 2013; 39:269-78.

11. Edgar D, Brereton M. Rehabilitation after burn injury. BMJ 2004; 329:343-5.

12. Dellinger RP, Levy MM, Rhodes A. Surviving sepsis campaign: International guidelines for management of severe sepsis and septic shock, 2012. Intensive Care Med 2013; 39:165-228.

13. Mandal P, Sidhu MK, Kope L. A pilot study of pulmonary rehabilitation and chest physiotherapy versus chest physiotherapy alone in bronchiectasis. Respir Med 2012; 106:1647-54.

14. Okhovatian F, Zoubine N. A comparison between two burn rehabilitation protocols. Burns 2007; 33:429-34.

15. Yang M, Yan Y, Yin X, Wang BY, Wu T, Liu GJ, et al. Chest physiotherapy for pneumonia in adults. Cochrane Database Syst Rev 2013; 2:CD006338. 